\title{
What Can You Do with a TaxonWorks API?
}

Matthew Yoder $^{\ddagger}$, Hernán Pereira§${ }^{\S}$, José Luis Pereiral, Dmitry A Dmitriev, Geoffrey Ower ${ }^{\ddagger}$, James R Flood $^{\ddagger}$

‡ University of Illinois at Urbana-Champaign, Champaign, Illinois, United States of America

§ División Entomología, Museo de La Plata, CEPAVE-CONICET, La Plata, Argentina

| División Entomología, Museo de La Plata, Universidad Nacional de La Plata, La Plata, Argentina

Corresponding author: Matthew Yoder (diapriid@gmail.com)

Received: 30 Sep 2020 | Published: 09 Oct 2020

Citation: Yoder M, Pereira H, Pereira JL, Dmitriev DA, Ower G, Flood JR (2020) What Can You Do with a

TaxonWorks API? Biodiversity Information Science and Standards 4: e59170.

https://doi.org/10.3897/biss.4.59170

\section{Abstract}

TaxonWorks is a web-based workbench facilitating curation of a broad cross-section of biodiversity informatics concepts. Its development is currently led by the Species File Group. TaxonWorks has a large, JSON serving, application programming interface (API). This API is slowly being exposed for external use. The API is documented at https:// api.taxonworks.org. Here we highlight some existing key features of the API focusing on the TaxonWorks concepts of People, Sources, Collection Objects, Taxon Names, and Downloads and provide a brief roadmap for upcoming additions. Highlights include the ability for data curators to produce shareable bibliographies, DarwinCore Archives (DwCA), and Catalogue of Life-formatted datasets, access their nomenclature as autocompletes and via many filter facets, share Person metadata including numerous identifier types, and perform basic Geo-JSON and simple DwC-A parameter-based filtering on Collection Objects. As examples of what can be done with the API, we provide several visualizations that are straightforward to implement by those with basic $\underline{R}$, Python, Javascript, or $\underline{\text { Ruby }}$ programming skills.

\section{Keywords}

JSON, script, curation, biodiversity informatics, workbench, digitization 


\section{Presenting author}

Matthew Yoder

\section{Presented at}

TDWG 2020 